\title{
Optimization of metallic X-ray capillary production
}

\author{
R. Mroczka ${ }^{\mathrm{a}}$, P. Bartosik ${ }^{\mathrm{a}}$, Z. Sawłowicz ${ }^{\mathrm{b}}$, K. Skrzypiec ${ }^{\mathrm{c}}$, G. Falkenberg $^{\mathrm{d}}$, J. Wójcik $^{\mathrm{c}}$, \\ G. Żukociński ${ }^{\mathrm{c}}$, A. Kuczumow ${ }^{\mathrm{a}, *}$ \\ a Department of Chemistry, KUL - The John Paul II Catholic University of Lublin, Al. Krasnicka 102, 20-718 Lublin, Poland \\ ${ }^{\mathrm{b}}$ Institute of Geological Sciences, Jagiellonian University, Cracow, Poland \\ ${ }^{\mathrm{c}}$ Marie Curie-Sklodowska University, Lublin, Poland \\ ${ }^{\mathrm{d}}$ HASYLAB, Germany
}

Available online 16 April 2008

\begin{abstract}
Among all of X-ray capillaries, those produced from metals attract special attention due to their specific advantages: less severe limitations on the value of the critical reflection angle, better control of the capillary shape, the maintenance of the straight main axis. The metallic, single bounce capillaries with gold and rhodium internal surfaces described in this paper are produced according to the original method invented at KUL. The production of the capillaries started from the formation of the internal steel mandrel of a designed shape that was later covered with another metal and finally pressed with the epoxy-resin. Then the mandrel was removed by the combination of mechanical and chemical actions. The shape of capillaries was controlled with the laser scan micrometer. The long-distance shape distortions, obeying so-called waviness correlation length were pointed out. The capillaries produced in our laboratory were characterized by the waviness amplitudes reaching $40-80 \mathrm{~nm}$ with correlation length about $300 \mu \mathrm{m}$. The symmetry of the opening and the straight shape of the main axis were investigated with the optical microscope and laser light transmitted through the capillary and registered with the CCD camera. The symmetry was found close to circular. The internal surface of the capillary was studied on the longitudinal cross-sections by means of the field emission scanning electron microscope (FESEM) and atomic force microscope (AFM). The surface roughness (rms) was determined, the parameter defining the ability of the surface to reflect X-rays efficiently in the total reflection mode. The best gold surfaces produced up-to-now had the rms $\sim 2 \mathrm{~nm}$, as measured on $1 \mu \mathrm{m} \times 1 \mu \mathrm{m}$ areas. The results of preliminary exercises with synchrotron radiation were demonstrated.
\end{abstract}

(C) 2008 Elsevier B.V. All rights reserved.

Keywords: X-ray capillary optics; Capillary production; Capillary diagnostics

\section{Introduction}

The modern applications of the X-rays demand the use of narrow pencil-like beams in many instances. The desired diameter of the beam is in the range from submicrons to a few hundreds of microns. Unfortunately, the primary X-ray sources, except a synchrotron, are as a rule the whole space or wide angle emitters. The technical solutions leading to obtaining the narrow beams are rather scarce, and involve, e.g., compound refractive lenses [1,2], Bragg-Fresnel plates [3,4], multicapillary semi-lenses [5] and single capillaries. The essential difficulties in focusing or only squeezing the beam result from the strict proximity of the X-ray refraction index value to the lower limit of a unity. The possibility

\footnotetext{
* Corresponding author. Tel.: +48 814454625 .

E-mail address: kuczon@kul.lublin.pl (A. Kuczumow).
}

of efficient reflection occurs exclusively in the small angle regime. The single capillaries are the simplest solution among above mentioned devices. They do not demand the coherent beam, hence any conventional source of X-rays could be employed without limitations. The single capillary device is well elaborated from the theoretical point of view [6,7]. Nevertheless, it is very difficult to produce a capillary of a small interior diameter with the fully controlled figure error, waviness and roughness of the surfaces and manufactured from the material with optimal mechanical and optical properties. Up to now, three kinds of materials were used for the production of capillaries: glass [8,9], metals and glass covered with metal. The metallic capillaries have some advantages: the greater values of the critical angles, which allowed the passage of the greater part of the wide primary beam through capillary; much easier control of the main axis straightness and the better performance during the 
heat overload. The newer studies on capillaries are aimed at the preparation of the metallic capillaries with the ability of single bounce [10-13]. It is the retreat from the earlier efforts, where the scientists were fascinated with the possibility of multiple bounces and treated the capillaries as the waveguides, leading X-rays to the clear change of the beam direction with simultaneous squeezing of the pencil. The work on the production of metallic capillaries was advanced in several research centers [14]. Our up-to-now contribution to the field was summarized in the previous paper [15].

The aim of this paper is to outline the method of the production of the metallic capillaries with the strictly designed shape and controlled waviness and roughness parameters. The chemical and geometrical stabilization of good quality internal surfaces of capillaries is necessary. The formation of the outer capillary wall with a minimum internal stress is one of the critical difficulties. The capillary diagnostics is another important question to be answered.

\section{Instrumental}

The electroplating operation was essential for forming the mandrel. Later on, the gold (rhodium) mirror layer was fixed and everything was covered with a body of the rigid capillary.

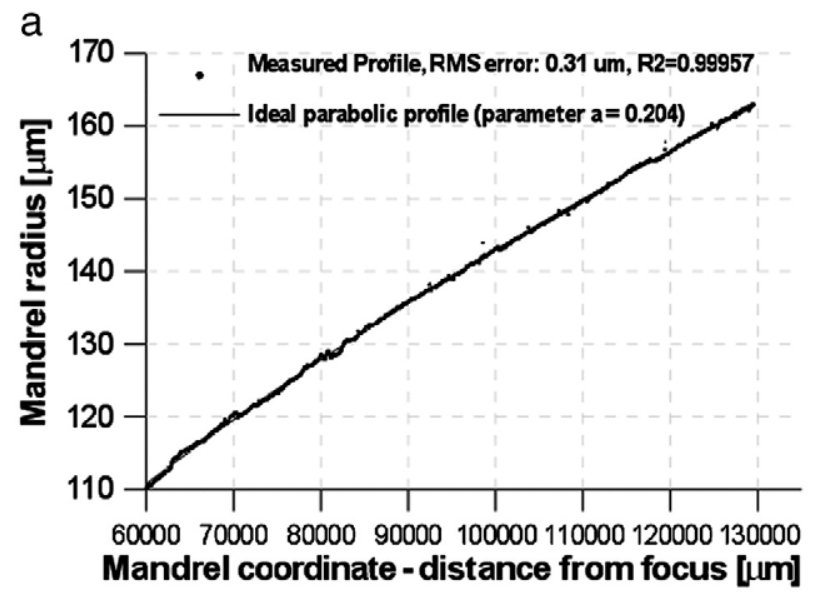

C

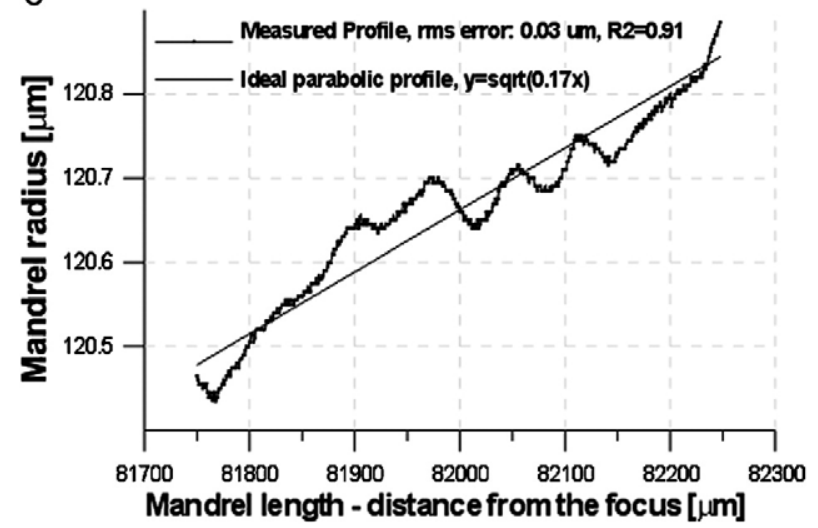

The process was carried out in an electroplating cell. The cell was equipped with the cylindrical grid anode made of $\mathrm{Ti} / \mathrm{IrO}_{2}$, of $47 \mathrm{~mm}$ diameter. The centrally positioned steel wire was a cathode. The galvanostatic unit EP-20 of ELPAN Company (Poland) assured the control of the current. The current density was set at about $20 \mathrm{mAcm}^{-2}$ at the beginning of the experiment. It fluctuated over time as the mandrel surface was changing. In the mandrel formation step, the controlled withdrawal of the cathode wire, being the center of the mandrel, from the solution was executed by the motorized stage PRIOR (England), computer steered. The copper layer was electrodeposited from the solutions of copper sulfate and sulfuric acid and additives such as: a surfactant, a brightener and a leveler, all at experimentally adjusted concentrations. The additives were essential and critical components. In order to improve the quality of the surface, the electropolishing process was carried out in the environment of phosphoric acid. This time, the mandrel was located as an anode. After polishing, the mandrel was covered with the thin gold (or rhodium) mirror layer in the electroless manner. Then the mandrel with the gold layer was coated either with a epoxy-resin or a thick, metallic electrodeposited layer. Finally, the core steel wire was removed by mechanic drawing and the copper part of the mandrel by the chemical etching.

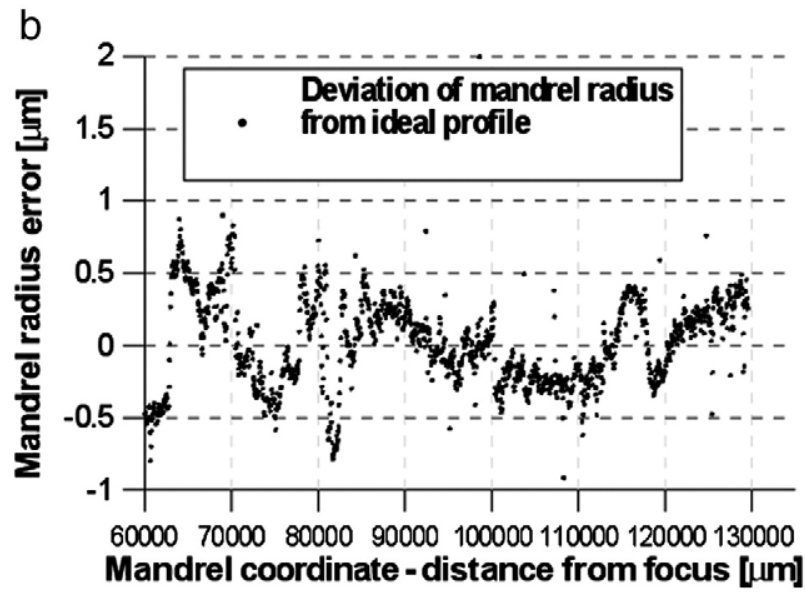

d

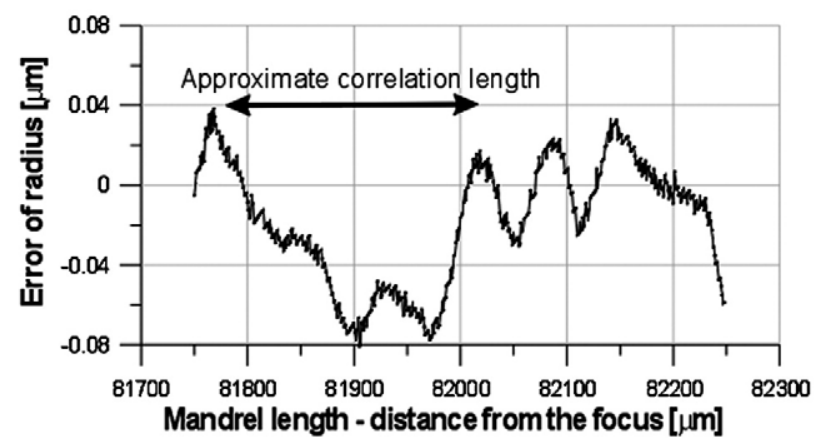

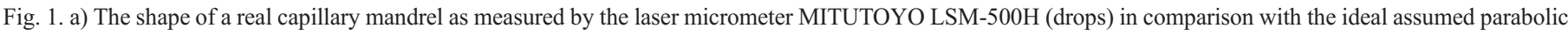

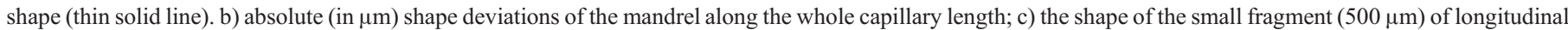

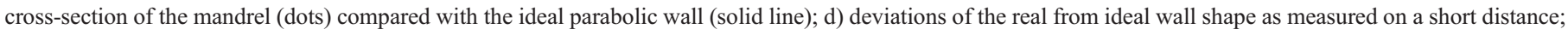
the arrows show the approximate correlation length. 
The mandrel transverse dimensions were measured by the laser scan micrometer MITUTOYO LSM-500H. The mandrel, located in the movable sample holder, was shifted before the laser optical track.

The field emission scanning electron microscope (FESEM) Hitachi S-4700 equipped with the energy dispersive $\mathrm{Si}(\mathrm{Li})$ detector EDX (Noran Vantage) at Laboratory of Field Emission Scanning Electron Microscopy and Microanalysis, Institute of Geological Sciences, Jagellonian University, Cracow, was employed for the observation of the mandrel surfaces, the interior of the capillaries and the capillary transverse crosssections. Some images were obtained using backscattered electrons (YAG detector). The accelerating voltage was set at $20 \mathrm{kV}$. In the cases where the electric charge was collected on the sample surface, the surface was covered with gold or graphite conductive layers. But the general tendency was to perform imaging and analyses of the raw samples. One of the aims was to compare the image of the surface from FESEM with the same image from AFM, in the same magnification, which was expected to deliver more comprehensive examination of the surface.

The roughness of the internal surface of the capillary was determined by means of the atomic force microscopy using NanoScope III, manufactured by Digital Instruments Co., USA. The measurements were performed in the contact mode of operation. Image processing software enabled the $2 \mathrm{D}$ and $3 \mathrm{D}$ surface presentation and the extraction of the histograms of the roughness along the selected lines. The instrument was at laboratories of University MCS in Lublin.

The experiments with some paraboloidal metallic capillaries were carried out in Line L at HASYLAB. The capillaries produced to fulfill the geometrical conditions of line (lengths in a range from 65 to $75 \mathrm{~mm}$ ) were introduced into capillary holder. The inlet parameters were in a range of $325-335 \mu \mathrm{m}$; for outlet $-215-230 \mu \mathrm{m}$. The beam divergence allowed by the internal shape was $4 \mathrm{mrad}$. The monochromatized beam with the photons with energies of $13 \mathrm{keV}$ was used. The beam was introduced in the normal inlet-outlet direction, but it was also inverted to compare the beam profile obtained in both directions (the first direction means focusing, the second the collimation). The beam output was collected in the focus position by the $\mathrm{CaWO}_{4}$ fluorescent screen and in far field by the CCD plate. Another way to check the beam profile was by mounting of the $4 \mu \mathrm{m}$ thick $\mathrm{W}$ wire in focus point and performing the linear scan in vertical direction with step of $1 \mu \mathrm{m}$. These measurements were carried out in two modes: firstly, when capillaries were mounted in normal position (synchrotron beam was entering into inlet side) and in inverted position (beam was entering to a tip with smaller diameter - outlet side). In the second case, capillary worked as usual collimator.

\section{Results}

Several tens of the capillaries with the assumed shapes were produced using the described technique. The thorough diagnosis of the capillaries was one of the most important tasks. The shape of the mandrel of a typical paraboloidal capillary, measured by

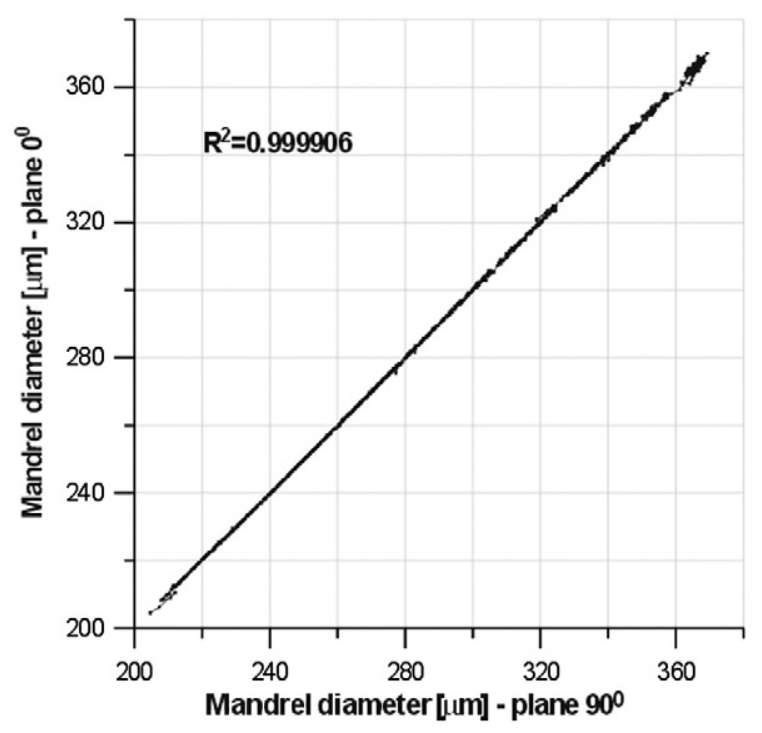

Fig. 2. Correlation diagram of the mandrel longitudinal shape determined without the rotation and with the rotation by $90^{\circ}$ — the proof of the rotational symmetry. The mandrel shapes were measured by the laser scan micrometer MITUTOYO LSM-500H.

the laser micrometer with the length resolution $50 \mu \mathrm{m}$, is shown in Fig. 1a. The absolute deviations of the real shape from the designed shape along the whole capillary length are presented in Fig. 1b. The small fragment of the mandrel (of the order of hundreds microns) was measured then with the length resolution $1 \mu \mathrm{m}$ to emphasize the real look of the waviness and more robust roughness - in a micrometer range, this image is shown in Fig. 1c. Hence the absolute deviations from the ideal shape could be measured on a distance of several hundreds of the micrometers along the capillary length and they are presented in Fig. 1d. The results presented in the figure and that obtained for other capillaries, not shown here, demonstrate some kind of mild waviness, with periods of the order of $300 \mu \mathrm{m}$ and amplitudes between $40-80 \mathrm{~nm}$. The comparison of Fig. $1 \mathrm{~b}$ and $\mathrm{d}$ shows that the deviations are nearly independent of the measured capillary length. These results can be used as a data base for the estimation of the waviness and robust roughness influence on the reflectivity.

Not only the longitudinal regularity should be estimated. In the similar way, the circular symmetry of the capillaries can be studied. The simplest tests comprise of consecutive scans after the rotation of the capillary by the assumed angle and comparison of the resulting curves presenting the longitudinal shapes of the wall. The correlation diagram between the shapes of the same mandrel measured in the initial position and rotated by $90^{\circ}$ is presented in Fig. 2. This result shows excellent correlation $\left(R^{2}=0.999906\right)$ for the perpendicular profiles, which indicates very good and repeatable circular symmetry, borrowed by the capillary from the symmetry of initial steel wire.

The procedure of electropolishing was applied for the optimal smoothing of the mandrel surface, that was previously put on the stainless steel wire. The surface of the wire before overbuilding, as observed by AFM, is shown in Fig. 3a. The optimal electropolishing time for the mandrel was determined 

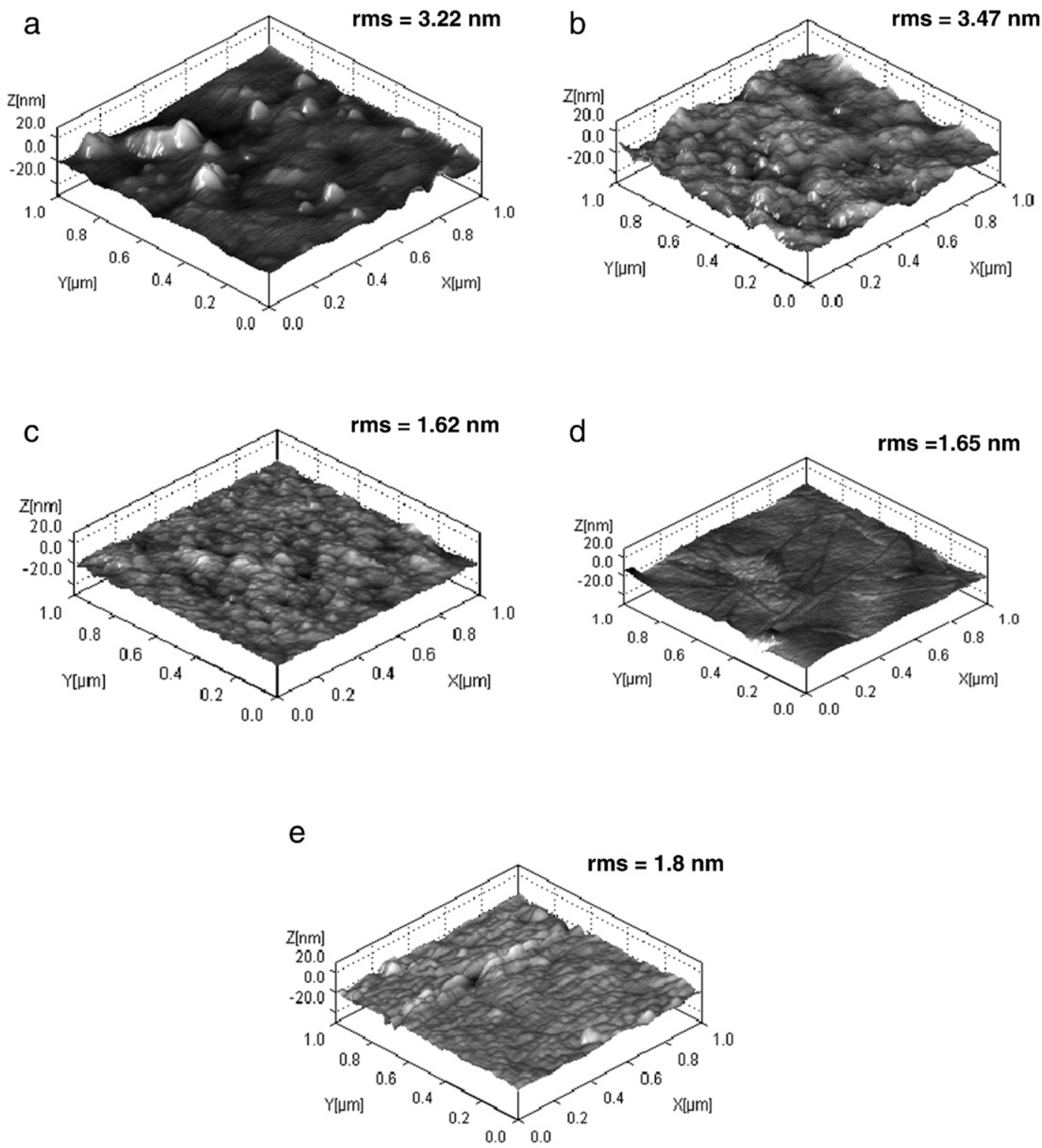

Fig. 3. a) The surface of the raw stainless steel wire; b) the raw surface of the copper mandrel; c) electropolished surface of the copper mandrel; d) the surface of the gold mirror interior of the capillary manufactured on the electropolished mandrel, close to the tip; e) the surface of the stainless steel wire improved with the passivation process. Measurements by the use of AFM, all mappings $1 \mu \mathrm{m} \times 1 \mu \mathrm{m}, X$ scale: $0.2 \mu \mathrm{m} /$ division., $Z$ scale: $100 \mathrm{~nm} / \mathrm{div}$. For Fig. $3 \mathrm{e} Z \mathrm{scale} 50 \mathrm{~nm} / \mathrm{div}$.

experimentally and it was in a range of $120-180$ s. During electropolishing, the rate of copper etching was about $1 \mu \mathrm{m} /$ min. When the process was carried out for a longer time, the small irreparable corrosion pits were formed. The typical effect of electropolishing, as observed by AFM, is shown in Fig. 3b and $\mathrm{c}$ for the surfaces before and after electropolishing, respectively and also presented in Table 1 . It is worth noting that we can improve the surface of the mandrel but we have no such possibilities for the glancing internal surface of the capillary. The observation of the mirror surface of the capillary by AFM is possible for some restricted areas (close to the capillary tip) and the typical result is shown in Fig. 3d. The mandrel for that capillary was electropolished. It seems that it exists some way of improving the process by increasing the quality of the primary stainless steel wire. It can be done by the passivation in the hot $20 \% \mathrm{HNO}_{3}$ solution. The resulting surface is shown in Fig. 3e.

The results of Table 1 suggest at first sight that the roughness is dependent on measured surface. It should be noticed that for each

Table 1

Rms (root mean square) of the roughness parameter (in [nm]) determined by AFM for the mandrel surface formed from the electrolytic bath

\begin{tabular}{llccc}
\hline & $\begin{array}{l}\text { Rms } \\
{[\mathrm{nm}]}\end{array}$ & $\begin{array}{c}\text { Surface size } \\
{[\mathrm{um} \times \text { um }]}\end{array}$ & $\begin{array}{c}\text { Rms } \\
{[\mathrm{nm}]}\end{array}$ & \\
\cline { 2 - 4 } Surface before polishing & 3.47 & $1 \times 1$ & 1.62 & \\
& 8.40 & $2 \times 2$ & 1.85 & Surface after polishing \\
& 8.96 & $5 \times 5$ & 2.27 & \\
& 11.28 & $10 \times 10$ & 2.78 & \\
& 12.75 & $20 \times 20$ & 4.73 & \\
\hline
\end{tabular}


a

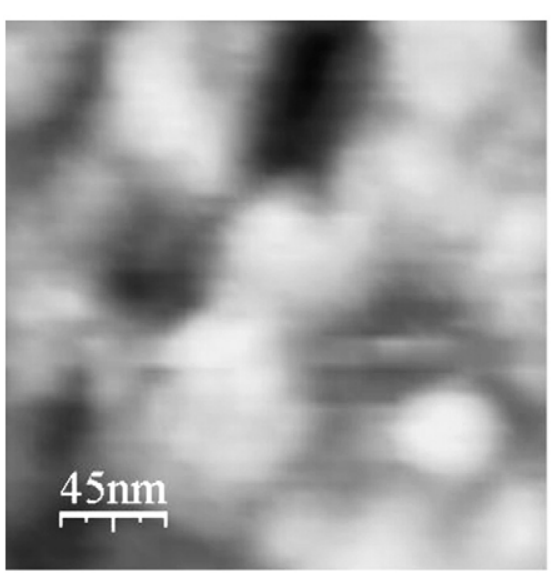

c

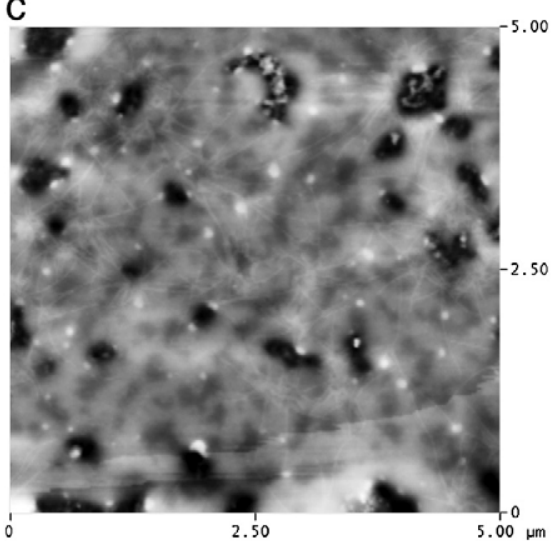

b

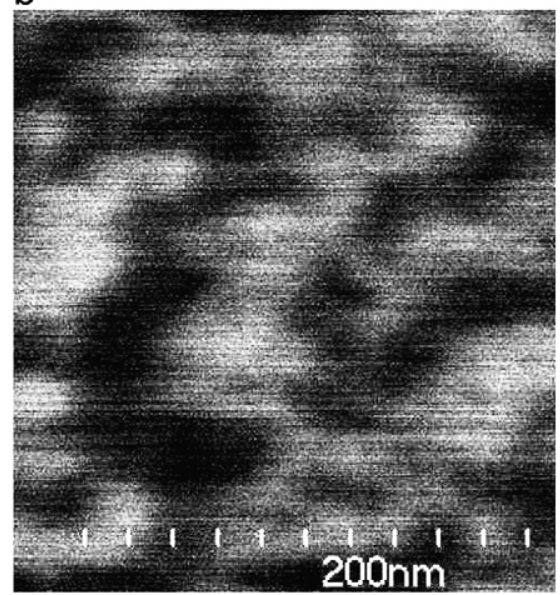

d

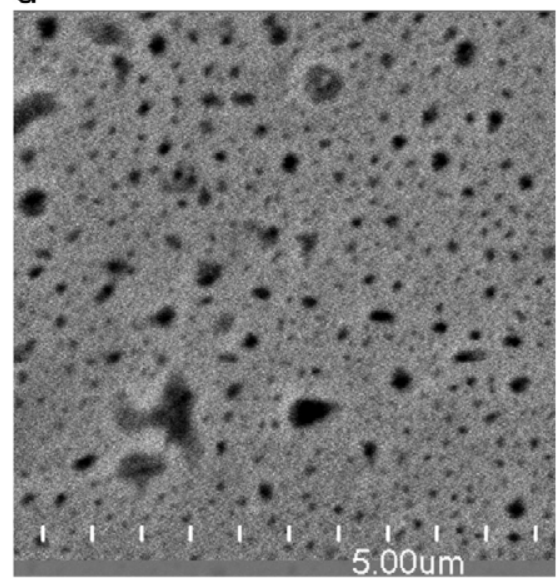

Fig. 4. Comparison of the images of the mandrel surfaces measured by: a) AFM and b) FESEM. Both images are $210 \times 215 \mathrm{~nm}$. Magnification for FESEM $200,000 X$. rms for AFM $-1.34 \mathrm{~nm}$. Comparison of the images of the interior of the glancing surface of the gold capillary as obtained by c) AFM and d) FESEM. Both images present the fields $5 \mu \mathrm{m} \times 5 \mu \mathrm{m}$. Magnification for FESEM $-10,000 X$. rms for AFM $-5.63 \mathrm{~nm}$.

measurement, the sampling step was different. For the smallest area, it was $4 \mathrm{~nm}$ and for the biggest area it was $78 \mathrm{~nm}$, respectively. Scaling analysis shows that roughness parameter $\sigma$ is saturated while measuring range is increased. In order to prove hypothesis of fractal nature of surface, additionally parameters should be determined: correlation length $\xi$, Hurst parameter (sometimes called roughness parameter) $\alpha$, skewness and kurtosis. All these parameters were found $(\alpha \sim 05, \xi \sim 50-300 \mathrm{~nm}$, depending on the surface area). It proves that surface of copper obtained in the electrodeposition process is isotropic and belongs to the self affine surface (it is special case of fractal surfaces).

Table 2

Rms (root mean square) of the roughness [nm] for the reflecting capillary surface formed with the electroless precipitation of gold

\begin{tabular}{ccc}
\hline & RMS $[\mathrm{nm}]$ & Surface $\operatorname{size}[\mu \mathrm{m} \times \mu \mathrm{m}]$ \\
\cline { 2 - 3 } M33 & 1.65 & $1 \times 1$ \\
& 3.45 & $2 \times 2$ \\
& 5.63 & $5 \times 5$ \\
& 6.45 & $10 \times 10$ \\
& 8.73 & $20 \times 20$ \\
\hline
\end{tabular}

The quality of the mandrel surface was examined by means of both AFM and FESEM. Due to the easy access to the mandrel surface, it was possible to collect backscatter electron images with the magnification 200,000X (Fig. 4b). Next to it, Fig. $4 \mathrm{a}$ shows the surface of the same capillary obtained by

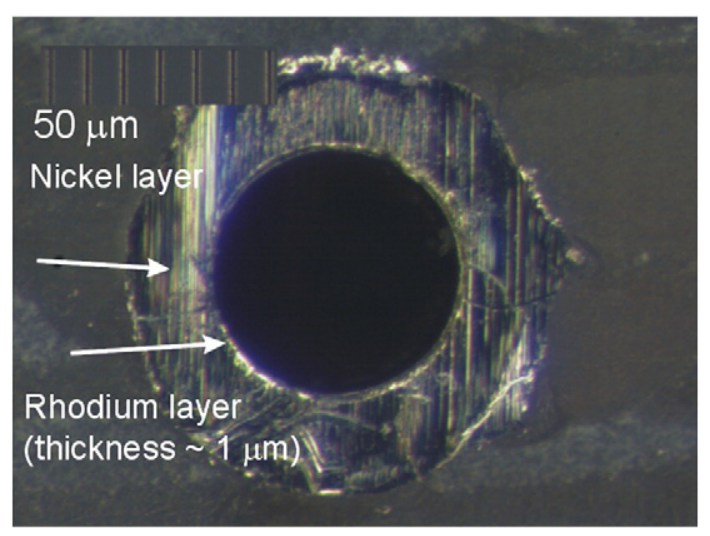

Fig. 5. Image of the capillary entrance cross-section recorded by the optical microscope. 


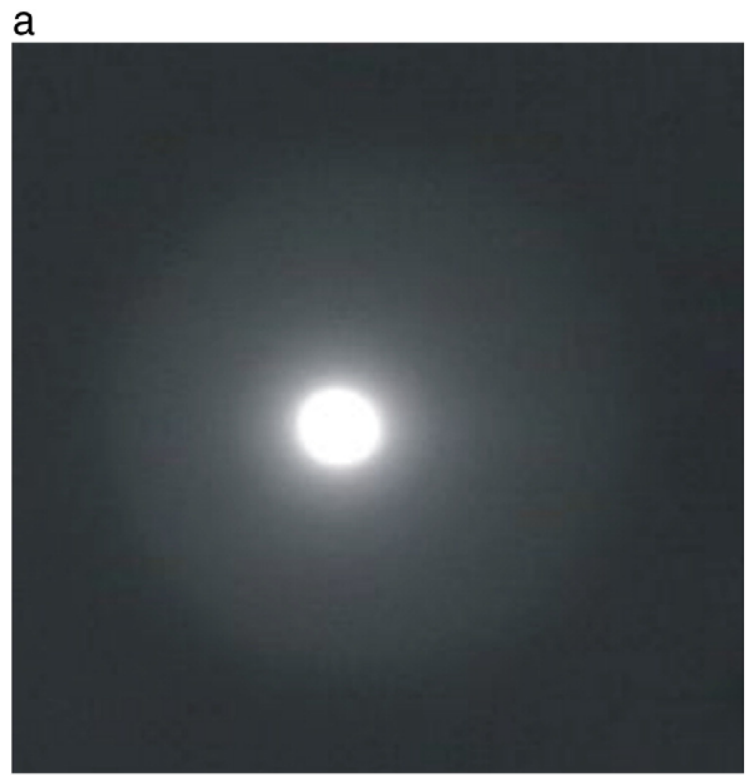

b

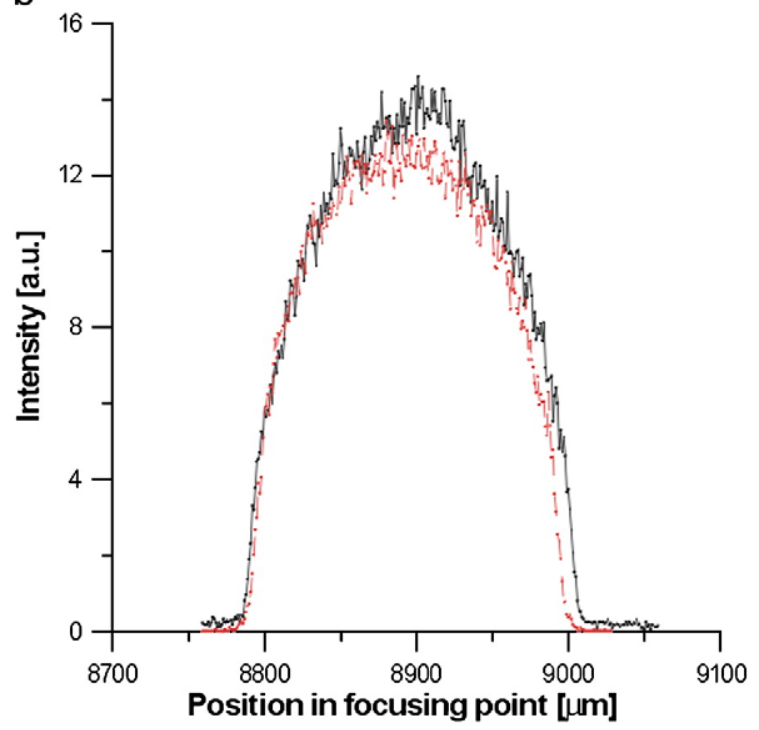

Fig. 6. a) Far field image of the beam passing through metallic capillaries with using synchrotron radiation of energy of $13 \mathrm{keV}$ at the HASYLAB L beam line. The beam profile is composed mainly from the direct contribution, without significant reflections; b) vertical profile of the beam recorded in focusing point ( $50 \mathrm{~mm}$ from the outlet tip of capillaries) by the scan with the tungsten wire after normalization (dotted line - beam profile recorded for capillaries in inverted position and solid one for capillaries mounted in normal position, respectively).

AFM imaging (the magnification in the figure was equalized to match that from FESEM). The tested localizations were not the same. At first sight, the essential morphological features of the surfaces seem to be uniform, which confirms the nonrandom, systematic character of the surface production.

In the same way, the internal reflecting surface of the capillary was studied. Both the AFM and FESEM measurements were applied again. The relevant mappings are shown in Fig. $4 \mathrm{c}$ and $\mathrm{d}$, respectively. It is worth noting that the measurements were again made for different positions in the capillary interior. In the case of AFM testing, the study was possible only for the very flat fragments on the capillary tips and another way of access to the interior was impossible. For FESEM measurements, the direct examination of nearly each location inside the interior of a cut off capillary was possible. Yet the concave surface of the capillary made the access difficult and the magnification of 10,000X was only possible. Nevertheless, good quality images were obtained. Despite different testing locations, the results were once again surprisingly uniform. We could observe the same kinds, sizes and numbers of the gold crystallites, e.g. greater ones with diameters around $0.2 \mu \mathrm{m}$ and smaller with diameters around $0.05-0.1 \mu \mathrm{m}$, similarly like in the case of mandrel surface. The result shows that electroless gold layer has a nearly amorphous structure with a number of very fine crystallites and it is a very accurate replica of the mandrel. The dependence of the rms on the size of the studied surface is presented in Table 2. The good compatibility between the rms for the mandrel and the capillary interior surface is observed, which indicates that the removal of the mandrel during the capillary forming does not worsen the quality of the internal surface. In that situation, the control of the mandrel surface is of essential significance as it defines the parameters of the glancing surface.

It was possible to control the inlet and outlet openings (Fig. 5) of the capillaries by the optical microscope, FESEM and indirectly by the registration of the transmitted laser light with the CCD camera. The image showing the cross-section of the capillary, shows the impressive circular symmetry and confirms fully the results showed earlier in Fig. 2.

This review is supplemented with the image of the X-ray beam profile from Line $\mathrm{L}$, after passing through the metallic capillary (Fig. 6a). No ring formed by reflected beam around direct beam is observed. Weak isotropic X-ray intensity around direct beam is caused by hallo effect. However, there is still some surplus of the beam intensity in the normal position of capillaries in comparison with the inverse direction and it testifies that the capillary is not an usual collimator or pin hole (Fig. 6b).

\section{Discussion}

The process of the metallic capillary production is relatively easy from the point of view of essential assumptions but more complicated in practice, with many troublesome technical details. As it was expected, the metallic capillaries, the production technology of which is totally different from the drawing technique used for the glass capillaries, show the significant improvement in keeping both the straight main axis and the circular symmetry on the cross sections. The obtaining of the assumed shape is also a strong feature of the technology. The working solid angle of these capillaries is significantly greater than their glass analogues with the same parameters of the shape. On the other hand, the internal roughness and periodic waviness of the internal glancing surfaces are still difficult to control. This problem can be overcome by comprehensive studying and optimization of process parameters. However, this is only true for the cases when the outer thick shell of the capillary does not experience internal stress. Otherwise, the stress relaxation leads to unpredictable displacements in the reflecting gold layer. It can increase especially the waviness error. At present, we are working 
on electroforming the outer capillary wall from nickel or copper electrolytic solution to eliminate this problem The control of the capillary shape was performed by the means of the scan laser micrometer. It is an alternative method to that used by Huang and Bilderback [13], who only measured the diameters of the external surface of the glass capillary with optical microscope and subtracted the thickness of a capillary wall from outer diameter, assuming the same ratio of the external to internal diameter for the whole length. Another method was used by Hirsch [14] for the metallic capillaries. He measured the diameters of the mandrel with the scanning electron microscope, but due to a non-ideally straight and flat shape of the mandrel, the errors in the diameter determination are inherent to this kind of measurement.

The methods of the surface diagnosis proposed for and employed in these studies delivered very credible and repeatable results - see Fig. 4 presenting the image of $0.2 \mu \mathrm{m} \times 0.2 \mu \mathrm{m}$ surface of the mandrel and $5 \mu \mathrm{m} \times 5 \mu \mathrm{m}$ region on the glancing surface of the capillary studied with the AFM and FESEM. The different locations on the same capillary were examined but the results were very similar. The large areas of very flat morphology broken with some splits in boundaries and covered with crystallites with typical sizes of 0.5 and $0.05 \mu \mathrm{m}$ can be observed for the metallic capillaries. The roughness parameter was determined for different studied areas, from $1 \mu \mathrm{m} \times 1 \mu \mathrm{m}$ to $20 \mu \mathrm{m} \times 20 \mu \mathrm{m}$. The results for the mandrels that were electropolished looked encouraging, the rms were below $5 \mathrm{~nm}$ for even largest surfaces studied with the AFM and often reached $1 \mathrm{~nm}$. Both the proper composition of the bath and the optimal time for the electropolishing operation for the mandrels were determined. However, the further tests are to be performed to identify if the parameters obtained are real limits for this stage. The roughness of the surface of primary steel wire transformed into the roughness of the mandrel, that in turn transformed into the roughness of the glancing surface of the capillary. It seems that the capillary production should start from the passivating of the stainless steel core to keep the roughness of this element at lowest possible level. Nevertheless, it appears that the roughness and especially waviness problems may be the most serious problems plaguing the metallic capillaries. The roughness rms determined for very good glass capillaries approach the values around $[16,17]$ or much below $1 \mathrm{~nm}[18]$ and still are lower than those obtained for the metallic capillaries. Similarly, the roughness parameters in the multilayer X-ray mirrors, decreased by ion polishing, were kept below $1 \mathrm{~nm}$ [19]. The poor performance of capillaries (they work nearly as usual collimator) arise from the irregularities of reflecting layer (the sum of these errors often is called slope error). The shape deviations (figure error) are very small and the roughness level is satisfactory (however, not such as in glass surfaces). From these two points, we can conclude that the waviness seems to be the main obstacle in good performance of the metallic capillaries. In our case, the amplitude is in a range of 40-80 nm and correlation length of waviness is too short (about $300 \mu \mathrm{m}$ ) that caused that the incidence angles were higher than the critical angle. It is confirmed by our calculations for capillaries [20]. Similar conclusions was also obtained by another researchers for capillaries [21] (waviness for glass capillaries was considered as surface distortion with $150 \mathrm{~nm}$ amplitude and a correlation length of $5000 \mu \mathrm{m}$ ) and for mirrors [22].

The electroless coating for the production of the mirror surfaces inside capillaries may be an alternative method to the vacuum deposition. Much lower cost, simple procedure and possibilities of deposition of much thicker (up to $5 \mu \mathrm{m}$ ) layers are the unquestionable advantages of the electroless procedures. However, the basic problem at that moment, is too high frequency undulation of surface (waviness) produced in elecrodeposition process (it probably arises from the electrochemical noise). This stage of our technology should be improved.

A significant progress in the analytical determination of the influence of this part of the roughness that is in a range of micrometer sizes and also the waviness on the reflectivity losses was made. The analytical procedures proposed proved to be successful in collecting and delivering necessary information.

\section{Acknowledgements}

The synchrotron measurements at HASYLAB were supported by the Contract II-05-006EC of the European Commission.

\section{References}

[1] A. Snigirev, V. Kohn, I. Snigireva, B. Lengeler, Nature 384 (1996) 49.

[2] A. Snigirev, V. Kohn, I. Snigireva, A. Souvorov, B. Lengeler, Appl. Opt. 37 (1998) 653.

[3] V.V. Aristov, Y.A. Basov, A.A. Snigirev, Rev. Sci. Instrum. 60 (1989) 1517.

[4] A. Snigirev, V. Kohn, in: W. Yun (Ed.), X-Ray Microbeam Technology and Applications, Proceed. SPIE, vol. 2516, 1995.

[5] M.A. Kumakhov, F.F. Komarov, Phys. Rep. (Rev. Sect. Phys. Lett.) 191 (1990) 289.

[6] L. Vincze, K. Janssens, F. Adams, X-ray Spectrom. 24 (1995) 27.

[7] A. Kuczumow, S. Larsson, Appl. Opt. 33 (1994) 7928.

[8] D.J. Thiel, D.H. Bilderback, A. Lewis, Rev. Sci. Instrum. 64 (1993) 2872.

[9] R. Mroczka, G. Żukociński, A. Kuczumow, J. Alloys Compd. 382 (2004) 311.

[10] D.H. Bilderback, S.A. Hoffman, D.J. Thiel, Science 263 (1994) 201.

[11] E. Fontes, Proceed. First Internat. Developers Workshop on Glass Capillary Optics for X-ray Microbeam Applications, Cornell Univ., Oct. 1996.

[12] S.B. Dabagov, A. Marcelli, Appl. Opt. 38 (1999) 7494.

[13] R. Huang, D.H. Bilderback, J. Synchrotron Radiat. 13 (2006) 74.

[14] G. Hirsch, X-Ray Spectrom. 32 (2003) 229.

[15] R. Mroczka, G. Żukociński, A. Kuczumow, J. Alloys Compd. 401 (2005) 108.

[16] D.X. Balaic, K.A. Nugent, Appl. Opt. 34 (1995) 7263.

[17] J.M. Bennett, Appl. Opt. 15 (1976) 2705.

[18] S. Kaupp, H. Wätzig, Electrophoresis 20 (1999) 2566.

[19] E. Spiller, SPIE 1160 (1989) 271.

[20] R. Mroczka, Ph. D. Thesis, [in Polish].

[21] L. Vincze, K. Janssens, F. Adams, R. Rindby, P. Engstrom, Rev. Sci. Instrum. 69 (1998) 3494.

[22] M. Sanchez del Rio, A. Marcelli, Nucl. Instrum. Methods, A 319 (1992) 170 . 